\title{
Entretien avec Olivia Loiseau
}

Mené par Michaël Abecassis, Université d'Oxford

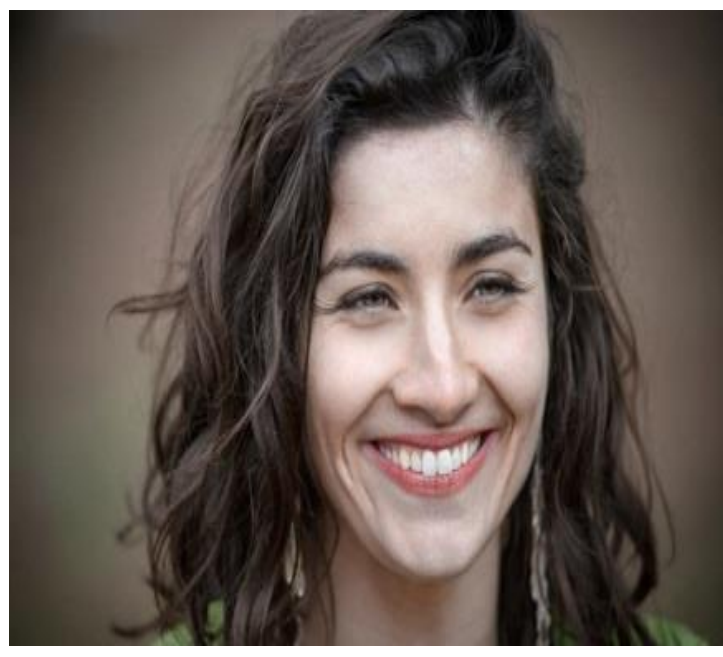

Olivia Loiseau est une actrice française née en Vendée. Après une formation de couturière, elle devient costumière pour des spectacles de danse de flamenco et de théâtre avant de se consacrer à l'art dramatique. Elle joue le rôle-titre dans le long métrage La sculpture vivante de Nguyen Tuong Hung et d'Aude Gourichon (monteuse/directrice de la photographie) qui sera projeté au Cinéma d'Art et d'Essai à Paris au Saint-André des Arts pour quatorze dates en février 2018. Elle joue également le rôle principal de Laura dans le courtmétrage INFRA Story de Fabrice Briseux projeté au Festival espagnol FICBUEU 2017.

(C) Philippe Brahim

Vous êtes une jeune actrice française qui a déjà joué dans de longs et courts métrages, comment en êtes-vous arrivée à vouloir faire du cinéma ?

Mes premières sensations dans l'amour du jeu ont commencé petite avec ma grande sœur, nous nous amusions à imiter les adultes qui eux-mêmes nous amusaient. J'avais ce réflexe aussi vers l'adolescence. Comme toute enfant, j'aimais mettre l'adulte face à lui-même dans ses comportements parce que je ne les comprenais pas toujours. Entre les blagues en famille et les sketchs qu'il y avait à l'école, j'ai eu l'envie de faire du théâtre à mes treize ans. J'ai eu aussi la chance d'avoir eu un beau-père très drôle qui me renvoyait souvent à rester dans la fibre artistique. Ayant peu confiance en moi à cette époque-là, je me suis tournée vers le métier de couturière/costumière car j'avais un goût prononcé pour les métiers manuels, de précision, jouant des couleurs et en lien avec le monde du spectacle. En parallèle de ma pratique du flamenco pendant sept ans, ma recherche dans la compréhension psycho/sociologique et émotionnelle de l'être humain, j'ai compris par le langage du corps et le personnage costumé que mes premiers pas dans le monde artistique me poussaient à faire du théâtre et du cinéma pour de bon, de réaliser ce rêve inavoué. Être actrice par vocation et par passion. 


\section{Comment choisissez-vous vos rôles ?}

J'aime sentir « la rencontre » avec le personnage, « la recherche » à travers le scénario ou le texte que je lis, son histoire qu'il se raconte à lui-même et que je peux entendre. Je suis beaucoup dans le ressenti et le fait de vouloir me dépasser moi-même à travers des événements vécus par des référents de notre histoire, par mon entourage ou par moi-même. La rencontre aussi avec l'équipe de travail est très importante, car c'est avant tout une rencontre humaine basée sur un partage essentiel pour réaliser un film.

\section{Se prépare-t-on différemment pour jouer un court-métrage et un long-métrage ?}

Je dirai qu'il y a des étapes de préparation qui divergent au fur et à mesure des circonstances de l'histoire du personnage durant le tournage... Me concernant, ma préparation est aussi importante pour un court-métrage que pour un long pour la bonne raison que l'envoi d'un message de l'acteur quel qu'il soit peut être aussi fort dans une durée limitée que pendant quatre-vingt-dix minutes. On m'a appris qu'il y a plusieurs méthodes de préparation différentes selon les acteurs, donc à partir du moment où vous êtes préparé à faire un court ou un longmétrage c'est à vous de bien savoir vous gérer dans le temps, de savoir que la disponibilité physique relatant aux faits demande plus d'effort souvent quand c'est un long-métrage. Dans un tournage, il y a des hauts, des bas, quelle que soit la durée du film. C'est souvent aussi l'implication de l'équipe du film et en fonction des moyens financiers qui sont investis que vous pouvez sentir plus d'exigence.

\section{Que pensez-vous du cinéma francophone?}

L'histoire du cinéma français est d'une grande richesse pour les acteurs car à travers son parcours, il me fait toujours rêver. Ses valeurs sont fortes et ne peuvent mourir du jour au lendemain. Le cinéma actuel se veut d'être ouvert au monde et c'est ce qui fait son évolution. Je pense que, quels que soient les moyens financiers dont il a besoin pour continuer à faire des films, il veut résister aux difficultés qu'il peut rencontrer dans le monde audiovisuel, le pouvoir de l'image étant tellement fort dans la vie des êtres humains. Ce que je ressens dans le cinéma français actuel, c'est une envie de liberté de création à travers tous les jeunes réalisateurs de demain qu'il s'empresse d'exprimer. Il est en train en ce moment de s'exporter grâce à de nombreuses productions, grâce à l'ouverture de nouveaux festivals. Je pense que c'est un élan très prometteur, évident pour toute une équipe de travail et reconnaissant de l'histoire du cinéma. 


\section{Quelle spectatrice êtes-vous ?}

Je suis assez réactive aux émotions que me renvoie une scène ou tout simplement un son fort ou subtil. J'aime sentir la communication avec les autres spectateurs dans la salle car l'atmosphère est importante quand vous vous préparez à regarder un film. Sensible à divers sujets, je porte souvent mon attention aux messages, aux faits, aux intentions des personnages et des réalisateurs plus qu'à la forme de l'image.

\section{Quel est le film qui vous a le plus marqué ?}

Il y en a beaucoup. Elephant Man de David Lynch m'a beaucoup marqué, je l'ai vu à l'âge de dix ans. Je peux vous citer Se souvenir des jolies choses avec Isabelle Carré, celle qui m'a donné l'envie aussi de faire ce métier, et Bernard Campan, une sensibilité mélangée à une insolence comique. L'important c'est d'aimer avec Romy, Green Snake de Tsui Hark d'une grande naïveté teintée de spiritualité, Tout sur ma mère de Pedro Almodovar, Les enfants du paradis de Marcel Carné, La vie de David Gale avec Kate Winslet de Alan Parker, The Reader de Stephen Dalry, Lady Chatterley de Pascale Ferran, The Straight Story, Mulholland Drive et la série Twin Peaks de David Lynch, Esprits rebelles de John N. Smith et d'autres...

En Angleterre, les acteurs passent facilement du théâtre au cinéma, mais en France les acteurs semblent se spécialiser soit dans l'un soit dans l'autre. Comment expliquez-vous cela?

Là encore, je dirais que tout dépend du parcours de chacun et de ses choix. Il y en a qui se sentent plus à l'aise au cinéma et d'autres au théâtre. Il y en a d'autres qui aiment entreprendre les deux, surtout si des opportunités se présentent dans leur carrière. Il faut bien rappeler que selon l'âge vers lequel vous commencez, le théâtre et/ou le cinéma, le temps est précieux et c'est par les choix que vous faites que vous dessinez votre carrière. Enfin pour d'autres, je pense que l'élocution du théâtre les attire moins. Peut-être que ce langage ne répond pas à leurs aspirations et leurs émotions profondes. L'écriture ancienne peut être incommodante et, à d'autres moments, attrayante selon la manière dont on l'aborde avec la nouvelle génération. A contrario, je connais de jeunes comédiens et comédiennes qui sont passionnés par le théâtre et ne souhaitent pas arrêter. Je peux dire aussi que la formation comédien/acteur diffère selon la recherche des réalisateurs. Certains préféreront des castings sauvages, former eux-mêmes leurs acteurs et d'autres aimeront ceux formés par le théâtre. On peut souligner le fait que le cinéma évolue peut-être à une cadence différente des formations classiques et de son enseignement. 
On ressent dans vos documentaires notamment dans La couturière votre passion pour la danse. Est-ce que la chorégraphie était storyboardée ou y a-t-il eu une grand part d'improvisation?

Pour La couturière, j'ai écrit le scénario à travers la musique, il n'y avait pas de story-board. Certaines scènes se devaient d'être improvisées.

Vos films semblent avoir un dénominateur commun : une recherche artistique voire esthétique. Est-ce que c'est la manière dont vous appréhendez vos rôles ou la manière dont le cinéma évolue?

Il y a des deux, car tout est lié à mon sens. L'art se veut d'être en perpétuelle évolution pour son goût de la nouveauté, de la découverte et de la spontanéité. Cependant, je ne tiens pas à ce que cette recherche me ferme à d'autres rôles pour le cinéma.

Comme le monde de la littérature où il existe une surabondance d'auteurs, au cinéma la demande est bien supérieure à l'offre ; comment arrive-t-on à se faire connaître et à percer dans ce milieu?

Je pense que le parcours de chacun est différent dans un premier temps. C'est une question de rencontres, de faire son réseau et surtout beaucoup de travail me concernant à fournir pour prouver ma valeur et mon amour du cinéma. Avant d'être actrice, j'avais une formation de couturière et puis je me suis beaucoup investie sur un projet culturel présentant l'art et le cinéma de David Lynch rencontré par la suite à Paris il y a deux ans. Vous savez, j'ai encore beaucoup à prouver, à apprendre ; c'est d'ailleurs aussi pour cette raison que j'aime ce métier, vous apprenez sans cesse et vous ne vous ennuyez jamais, c'est un bon moteur de travail à mon sens. Suivez votre intuition et l'énergie suivra.

\section{Vous avez fait de la voix off. Comment travaille-t-on sa voix pour cet exercice ?}

J'ai prêté ma voix pour de la musique d'ambiance de l'album «Diaries» Chemical Dysfunction réalisé par le compositeur Londonien Frank Cronogue, et aussi pour un entretien critique des fans du cinéma de David Lynch, pour Les mémoires de la marquise de Bonchamps, femme du militant Charles Melchior Artus de Bonchamps, un des commandants et héros de l'armée vendéenne du temps de la révolution française. A proprement parler, je n'avais pas de technique spécifique pour ces exercices étant donné que c'était un mélange de théâtre et de mélodies selon les projets. Il s'avère que l'enregistrement de ma voix pour Frank s'est fait à distance. De ce fait, j'échauffais ma voix et répétais plusieurs fois les textes qui m'étaient 
envoyés. Néanmoins, les cours de chant m'ont permis de faire quelques exercices au préalable, surtout que le travail de narration pour Les mémoires de la marquise de Bonchamps était assez long et intense pour ma voix.

Le court métrage Infra-story traite de la relation de deux femmes que trente-cinq années séparent et de la liberté d'aimer, mais il y a aussi toute une dimension poétique liée à la peinture. Quel souvenir de tournage gardez-vous de ce film et est-ce qu'il y a quelque chose d'impudique à révéler ses émotions à l'écran?

Le souvenir que je garde est une équipe très impliquée dans le projet de ce film dont certains travaillaient dans une autre branche. L'ambiance était très conviviale et nécessaire pour ce film au sujet délicat. Le souvenir que je garde en mémoire est le moment où l'on a tourné de nuit près d'un ruisseau, c'était une grande sensation car cette scène évoquait un rêve/cauchemar onirique. Je n'ai pas ressenti de pudeur en exprimant mes émotions à la caméra car je sers un personnage et non moi-même.

Quand on vit l'intensité d'un tournage est-il facile de passer à un autre film et à une autre équipe sans se sentir affecté par cette rupture?

Me concernant cela ne m'est jamais arrivé, car pour moi le métier d'actrice est de jouer et d'être disponible pour l'équipe avec qui je travaille. Les traces de l'affect n'appartiennent qu'à vous et vous les portez comme de beaux souvenirs.

A la fin de sa vie, Rodin s'est beaucoup interrogé sur la matière vivante en essayant notamment de donner l'apparence de la vie à travers ses marbres. Est-ce que la recherche perpétuelle de l'artiste dans La sculpture vivante pour sublimer la mort est la quintessence même du film ?

Un film est un ensemble d'images et de miroirs pour l'acteur et le spectateur. Les Anciens disent que la vie est un spectacle permanent, je le pense aussi. En ce sens, la sublimité de la mort se vit en toute chose, c'est le cas pour l'artiste Michel Angelo. Sa soif d'amour à son âge bien avancé est tellement intense que ses gestes ne demandent qu'à sublimer ce qu'il peut voir à travers les personnages rencontrés. L'image à l'écran n'est qu'un vecteur d'intensification liée à une manifestation. Pour moi, il est fondamental d'utiliser l'image pour servir la vie audelà de tout, d'où ma recherche de la quintessence d'une œuvre / d'un scénario. 


\section{Quel est votre rêve d'actrice?}

Mon rêve est de pouvoir avoir l'opportunité de tourner à l'étranger tout en persévérant dans le cinéma français et dans mes activités en parallèle.

\section{Est-ce que le fait d'être actrice vous donne un regard différent sur la vie ?}

Quand on est acteur ou actrice, on se doit d'avoir un esprit ouvert et clairement disponible à ce qui nous entoure, c'est de la nourriture pour le jeu. De ce fait, le regard doit se permettre d'explorer les variations de l'esprit dans son univers de spectateur/acteur. Lorsque j'ai commencé le théâtre, mon regard observait drôlement le monde et ses énergies d'une manière différente pendant un instant. À travers le temps, à mon sens, c'est un ensemble de choses que vous vivez qui peut vous amener à changer de regard sur la vie, l'image n'étant que le témoin de notre existence et de nos émotions. A contrario, une scène regardée au cinéma peut tout aussi bien vous apporter une compréhension nouvelle dans un cours instant sur des questions existentielles que vous vous posez. Par l'image, j'ai compris que toute forme de pensée, de manifestation, d'action ou d'émotion nous ramène sans cesse à comprendre que la vie « est à la fois tout et son contraire ». Plus petite, je regardais le monde de manière individuelle, de façon à me construire mes propres opinions puis, dans la maturité, à remanier ma pensée par des échanges avec les autres et par des explorations...

\section{Quel est votre meilleur souvenir de tournage?}

Le meilleur souvenir de tournage que je retiens, c'est le dernier jour tourné pour le film $L a$ sculpture vivante, je ne peux pas en dire plus... 